\title{
Elliptical heads result in increased glenohumeral translation along with micro-motion of the glenoid component during axial rotation in total shoulder arthroplasty
}

\author{
Lukas N. Muench ${ }^{1,2}{ }^{(1)} \cdot$ Cameron Kia $^{1} \cdot$ Matthew Murphey ${ }^{1} \cdot$ Elifho Obopilwe $^{1} \cdot$ Mark P. Cote $^{1} \cdot$ Andreas B. Imhoff $^{2}$. \\ Augustus D. Mazzocca ${ }^{1}$ Daniel P. Berthold ${ }^{1,2}$
}

Received: 19 January 2021 / Accepted: 23 June 2021 / Published online: 3 July 2021

(c) The Author(s) 2021

\begin{abstract}
Introduction Elliptical-shaped humeral head prostheses have recently been proposed to reflect a more anatomic shoulder replacement. However, its subsequent effect on micro-motion of the glenoid component is still not understood.

Materials and methods Six fresh-frozen, cadaveric shoulders (mean age: $62.7 \pm 9.2$ years) were used for the study. Each specimen underwent total shoulder arthroplasty using an anatomic stemless implant. At $15^{\circ}, 30^{\circ}, 45^{\circ}$ and $60^{\circ}$ of glenohumeral abduction, $50^{\circ}$ of internal and external rotations in the axial plane were alternatingly applied to the humerus with both an elliptical and spherical humeral head design. Glenohumeral translation was assessed by means of a 3-dimensional digitizer. Micro-motion of the glenoid component was evaluated using four high-resolution differential variable reluctance transducer strain gauges, placed at the anterior, posterior, superior, and inferior aspect of the glenoid component.

Results The elliptical head design showed significantly more micro-motion in total and at the superior aspect of glenoid component during external rotation at $15^{\circ}$ (total: $P=0.004$; superior: $P=0.004$ ) and $30^{\circ}$ (total: $P=0.045$; superior: $P=0.033$ ) of abduction when compared to the spherical design. However, during internal rotation, elliptical and spherical heads showed similar amounts of micro-motion at the glenoid component at all tested abduction angles. When looking at glenohumeral translation, elliptical and spherical heads showed similar anteroposterior and superoinferior translation as well as compound motion during external rotation at all tested abduction angles. During internal rotation, the elliptical design resulted in significantly more anteroposterior translation and compound motion at all abduction angles when compared to the spherical design $(P<0.05)$.

Conclusion In the setting of total shoulder arthroplasty, the elliptical head design demonstrated greater glenohumeral translation and micro-motion at the glenoid component during axial rotation when compared to the spherical design, potentially increasing the risk for glenoid loosening in the long term.
\end{abstract}

Level of evidence Controlled Laboratory Study

Keywords Humeral head · Elliptical · Spherical · Prosthesis design · Total shoulder arthroplasty · Micro-motion · Glenohumeral translation

Lukas N. Muench

lukas.muench@tum.de

1 Department of Orthopaedic Surgery, UConn Health, Farmington, CT, USA

2 Department of Orthopaedic Sports Medicine, Technical University of Munich, Munich, Germany

\section{Introduction}

Although anatomic total shoulder arthroplasty (TSA) has been shown to provide sufficient pain relief and improvement in shoulder function for patients with end-stage glenohumeral osteoarthritis, glenoid component loosening has been reported to be one of the main contributors to postoperative implant failure $[6,19,23,26]$. With a prevalence varying widely between 27 and $94 \%$ of cases, peri-glenoid radiolucent lines have also been associated to poorer patientreported functional outcomes following TSA $[6,19,26]$. 
As previous studies have identified eccentric loading along with the resulting rocking of the glenoid component as an important biomechanical factor for implant loosening, the glenoid design has been suggested to be critical for long-term stability and clinical survival [2-4]. Biomechanically, Voss et al. recently found that a pegged glenoid design showed significantly increased micro-motion during eccentric axial loading when compared to a keeled glenoid design [29]. Although with this suggesting that the glenoid design is important for initial fixation strength, Throckmorton et al. did not find significant differences in clinical and radiographic outcomes between the two designs [28]. More importantly, this inconsistency may imply that other factors further influence the development of glenoid loosening, including the design of the humeral head prosthesis.

Recent literature has described the humeral head to be more elliptical in shape, rather than a perfect sphere [8, 11, 15]. As implants resembling the native anatomy may restore joint kinematics and ensure durability most sufficiently, the use of elliptical prosthetic head designs has become more popular $[5,13,17]$. In a dynamic shoulder model, elliptical and spherical heads demonstrated similar degrees of the total, internal, and external rotational range of motion (ROM) in shoulder arthroplasty [22]. However, a biomechanical study by Jun et al. found that non-spherical heads resulted in increased glenohumeral translation during axial rotation in the coronal, scapular, and forward elevation plane at various abduction angles when compared to spherical heads [18]. While an increased translation may resemble native kinematics more accurately, this may also lead to more eccentric loading on and greater micro-motion of the glenoid component, potentially resulting in implant failure over time.

Thus, the purpose of this study was to investigate if there would be a difference in glenohumeral translation and micromotion of the glenoid component during axial rotation when comparing elliptical and spherical prosthetic heads in the setting of TSA. The authors hypothesized that the elliptical prosthetic head design would result in significantly greater glenohumeral translation along with micro-motion of the glenoid component compared to the spherical head.

\section{Materials and methods}

Six fresh-frozen, cadaveric shoulders with a mean age of $62.7 \pm 9.2$ years (range $48-74$ years) were used for the study (Science Care Inc., Phoenix, AZ, USA). As de-identified specimens were not considered to constitute human subjects research, prior Institutional Review Board approval was not required.

\section{Specimen preparation}

After having been thawed overnight at room temperature, specimens were dissected free of skin, subcutaneous tissue, muscles and capsule. Following disarticulation, specimens underwent visual inspection to exclude those with moderate to severe osteoarthritis or bony defects. Under fluoroscopy control (Mini C-Arm, GE Medical Systems Inc.), a $2.0 \mathrm{~mm} \mathrm{~K}$-wire was drilled parallel to the glenoid surface from posterior to anterior at the middle of the superior-inferior diameter. A second $2.0 \mathrm{~mm} \mathrm{k}$-wire was drilled from inferior to superior parallel to the glenoid. The scapula body was trimmed using an oscillating saw and potted in a custom rectangular box with the glenoid surface being aligned parallel to the floor. The humerus was shortened and all soft tissues were completely removed. It was then centered and potted in a poly-vinyl chloride (PVC) pipe (diameter, $3.8 \mathrm{~cm}$; length, $7 \mathrm{~cm}$ ) using bone cement, leaving only $2 \mathrm{~cm}$ of the proximal humeral shaft exposed, to minimize diaphyseal bending moments $[16,20]$.

\section{Surgical technique}

All surgical procedures were performed by the same surgeon (L.N.M) to minimize performance bias. TSA was performed using an anatomic stemless implant (Eclipse system, Arthrex Inc., Naples, FL, USA) as previously described [7, 10]. Oriented along the specimen's anatomic retrotorsion, two $1.6 \mathrm{~mm} \mathrm{~K}$-wires were pre-drilled in line with the desired resection plane, exiting the opposite cortex at the boundary of the articular cartilage. Guided by the two K-wires, subsequent osteotomy was performed using an oscillating saw. After measuring the anterior-posterior dimension of the resected humeral head, the size of the baseplate (trunnion) was determined. The trunnion was then fixed to the resected humeral neck and a hollow screw was inserted. Additionally, the custom-made trunnion used for this study was secured with a small, protruding spike, to allow for easily switching the different prosthetic heads during testing.

Glenoid replacement was performed using a keeled glenoid system (Univers II, Arthrex Inc., Naples, FL, USA). A glenoid guide was placed on the central axis of the exposed articular surface of the glenoid, with the guide handle being oriented in line with the anatomic slope of the anterior neck. Following preparation, a keeled glenoid implant was inserted in the created slot and impacted.

\section{Humeral head prosthetic design}

Both elliptical and spherical prosthetic humeral heads were custom-made (Arthrex Inc., Naples, FL, USA). The designs, 
including equations for dimension width, radius of curvature, and height, were chosen according to previously published studies [13, 14]. A small hole in the under-surface allowed for securely placing the humeral head prosthesis on the protruding spike of the trunnion, avoiding rotation of the head prosthesis during testing and allowing for easily switching heads between testing conditions.

\section{Testing setup}

The specimens were mounted to a validated shoulder testing rig as previously described, which allowed for positioning of the glenohumeral joint in 6 degrees of freedom $[1,20$, $21,24,25]$. With the glenoid surface being in a horizontal position, the scapula was fixed to a vertical linear bearing translator and lever arm system on top of an $\mathrm{X}-\mathrm{Y}$ table, allowing for glenohumeral translation in the anteroposterior and superoinferior direction (Fig. 1). The rotation of the humerus was defined as neutral with the bicipital groove being aligned with the anterior margin of the acromion according to Selecky et al. [16, 27]. To determine micromotion of the glenoid component, four high-resolution differential variable reluctance transducer (DVRT) strain gauges (Microstrain, Burlington, VT, USA) were placed at the anterior, posterior, superior, and inferior aspect of the glenoid component (Fig. 2) [29].

\section{Biomechanical testing}

During testing, an axial compression load of $40 \mathrm{~N}$ was constantly applied via the lever arm of the $\mathrm{X}-\mathrm{Y}$ table to center the joint [18]. According to Jun et al. [18], 50 of internal and $50^{\circ}$ of external axial rotation were alternatingly applied
Fig. 1 With the glenoid surface being in a horizontal position, the scapula is fixed to a vertical linear bearing translator and lever arm system on top of an $\mathrm{X}-\mathrm{Y}$ table. The rotation of the humerus is in neutral position. To determine micro-motion of the glenoid component, four high-resolution DVRT (red boxes) are placed at the anterior, posterior, superior, and inferior aspect of the glenoid component. A Anterior view. B Inferior view
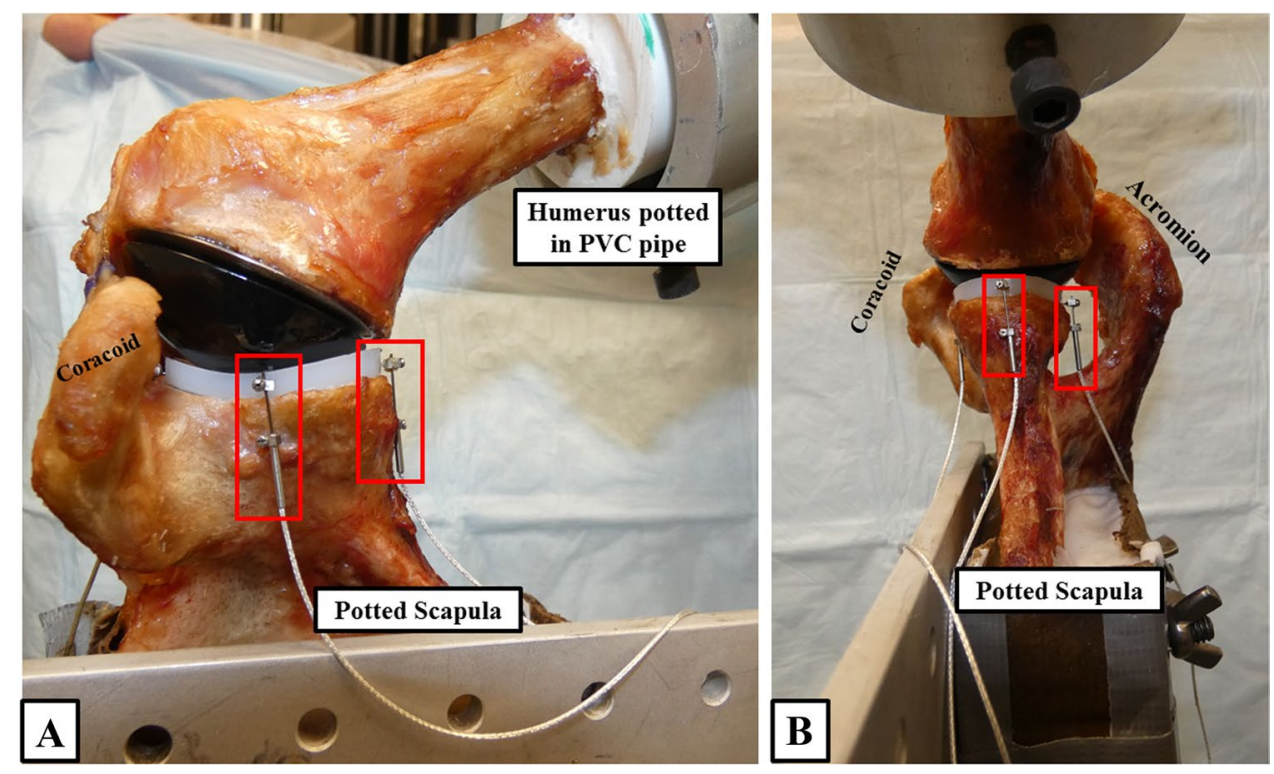

Fig. 2 To determine micromotion of the glenoid component, four high-resolution DVRT (red boxes) are placed at the anterior, posterior, superior, and inferior aspect of the glenoid component. A Bird's-eye view. B Anterior view
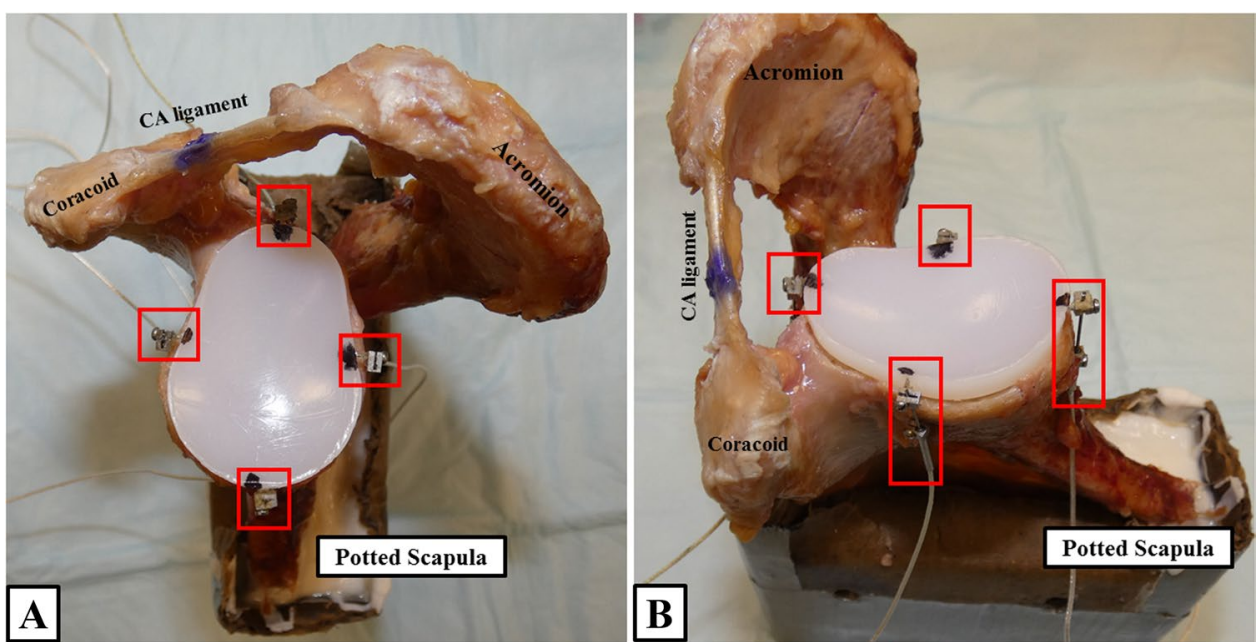
to the humerus at $15^{\circ}, 30^{\circ}, 45^{\circ}$, and $60^{\circ}$ of glenohumeral abduction in the scapular plane.

By means of a 3-dimensional digitizer (MicroScribe G2; Immersion) with a position accuracy of $0.23 \mathrm{~mm}$, the position of the $\mathrm{X}-\mathrm{Y}$ table was measured by carefully digitizing the center of a defined groove on the $\mathrm{X}-\mathrm{Y}$ table without relevant influence by touching off with the digitizer. The position of the groove was determined at the beginning (start position) and the end (end position) of each application of internal or external rotation. Changes in the position represented the glenohumeral translation and were given in anteroposterior ( $x$-axis) and superoinferior ( $y$-axis) directions. In addition, overall compound motion during internal and external rotation was calculated as the square root of the sum of the squared anteroposterior ( $x$-axis) and squared superoinferior ( $y$-axis) translation.

After completion of translational testing for each condition, micro-motion of the glenoid component during internal and external rotation was assessed using the mounted DVRT strain gauges. The final position in internal or external rotation was maintained for five seconds to allow the measuring curve to form a plateau for analysis.

During evaluation of translation and micro-motion, internal and external rotations were each applied five times for every condition. Values of each specimen were then averaged and are presented as the final values. Throughout the entire testing, specimens were not removed from the testing rig, nor was the testing rig disassembled. To avoid selection bias, the order of glenohumeral abduction positions $\left(15^{\circ}\right.$, $30^{\circ}, 45^{\circ}, 60^{\circ}$ ) and head designs (elliptical or spherical) was randomly assigned.

\section{Statistical analysis}

A power analysis was carried out to determine detectable differences in micro-motion, using standard deviations estimated from the literature as well as pilot data prior to this study [29]. Assuming a common standard deviation of
$0.1 \mathrm{~mm}$, a sample size of 6 specimens would provide $80 \%$ power to detect a $0.15 \mathrm{~mm}$ difference in micro-motion at an $\alpha$ level of 0.05 .

Differences in micro-motion and translation between implants were assessed using multilevel mixed effects generalized linear models. A random intercept was used to account for specimens in different conditions. The gamma distribution was used to model micro-motion. For each analysis, the distribution of the residual was examined and found to conform to a normal distribution. Comparisons of marginal mean values were carried out and were adjusted for multiple comparisons using the Holm-Bonferroni sequential correction method in the presence of initial statistical significance. A $P$ value of 0.05 was set to be statistically significant. All statistical analyses were conducted using Stata 15 software (StataCorp. 2017. Stata Statistical Software: Release 15. College Station, TX: StataCorp LLC).

\section{Results}

\section{Micro-motion of the glenoid component}

The elliptical humeral head design showed significantly more micro-motion in total (Fig. 3) and at the superior aspect of glenoid component during external rotation at $15^{\circ}$ (total: $P=0.004$; superior: $P=0.004$ ) and $30^{\circ}$ (total: $P=0.045$; superior: $P=0.033$ ) of abduction when compared to the spherical design (Table 1). Further, elliptical heads resulted in significantly more micro-motion at the posterior aspect of the glenoid component during external rotation in $15^{\circ}$ of abduction $(P=0.004)$. However, there was no significant difference in micro-motion at the inferior and anterior aspects of the glenoid ( $P>0.05$, respectively). In addition, there were no significant differences in micro-motion during external rotation for both elliptical and spherical heads when comparing the resting position $\left(15^{\circ}\right)$ to the abduction angles of $30^{\circ}, 45^{\circ}$, and $60^{\circ}(P>0.05$, respectively $)$.
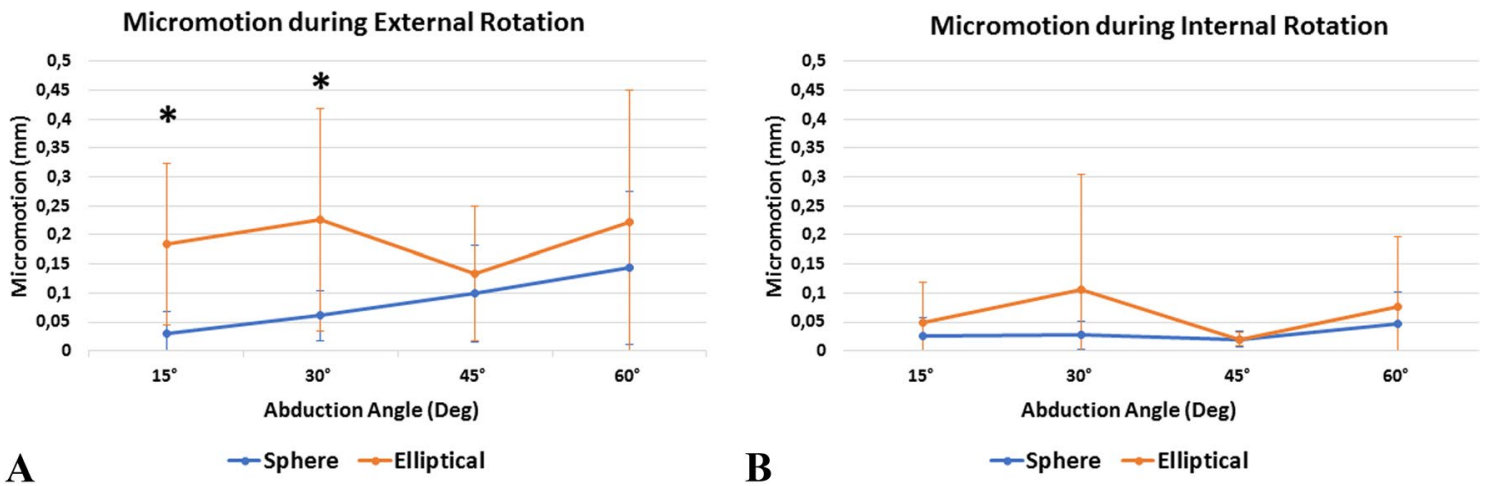

Fig. 3 Total micro-motion (mm) at the glenoid component during external (A) and internal (B) rotation. * Indicates statistical significance 


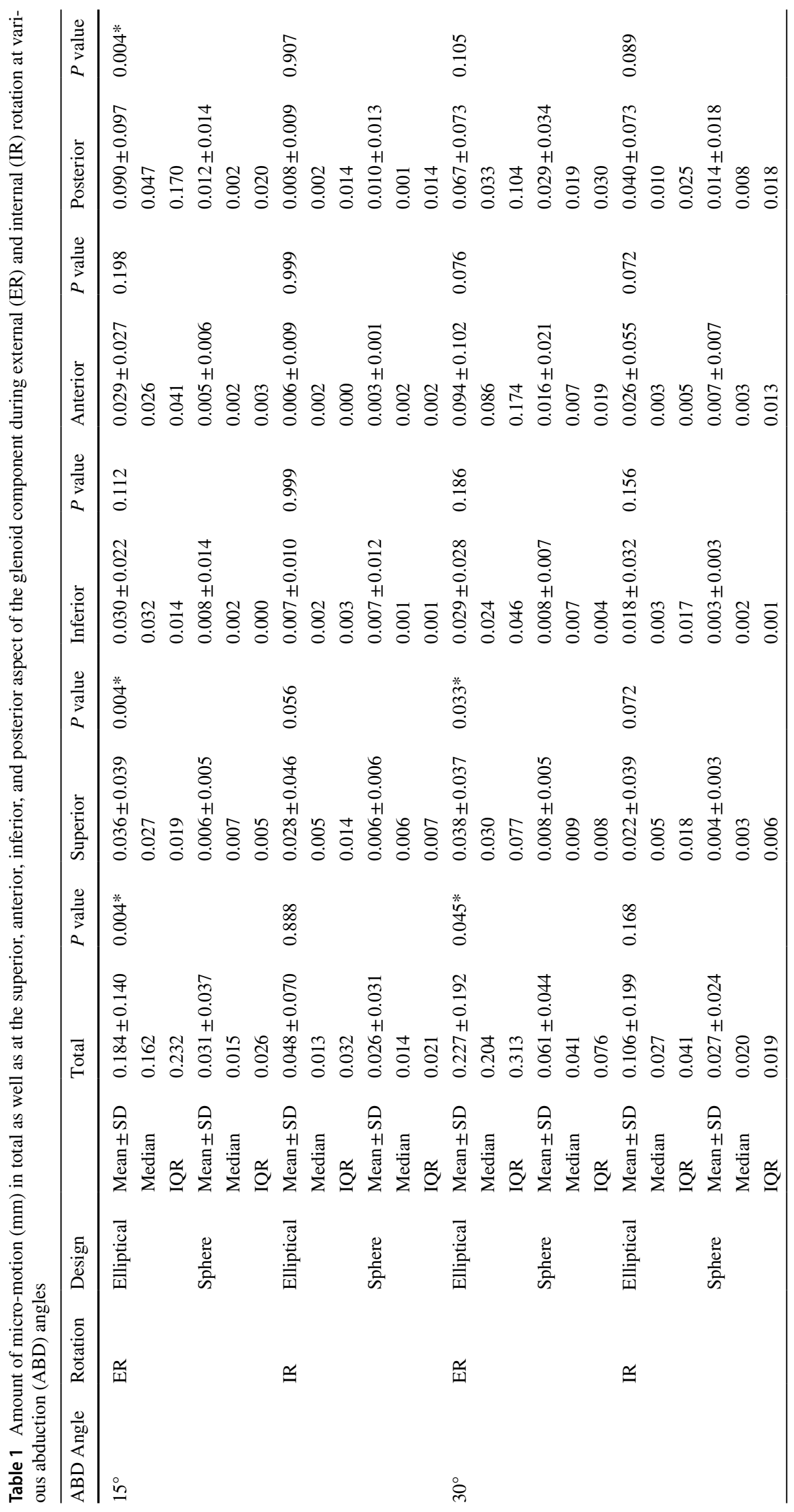




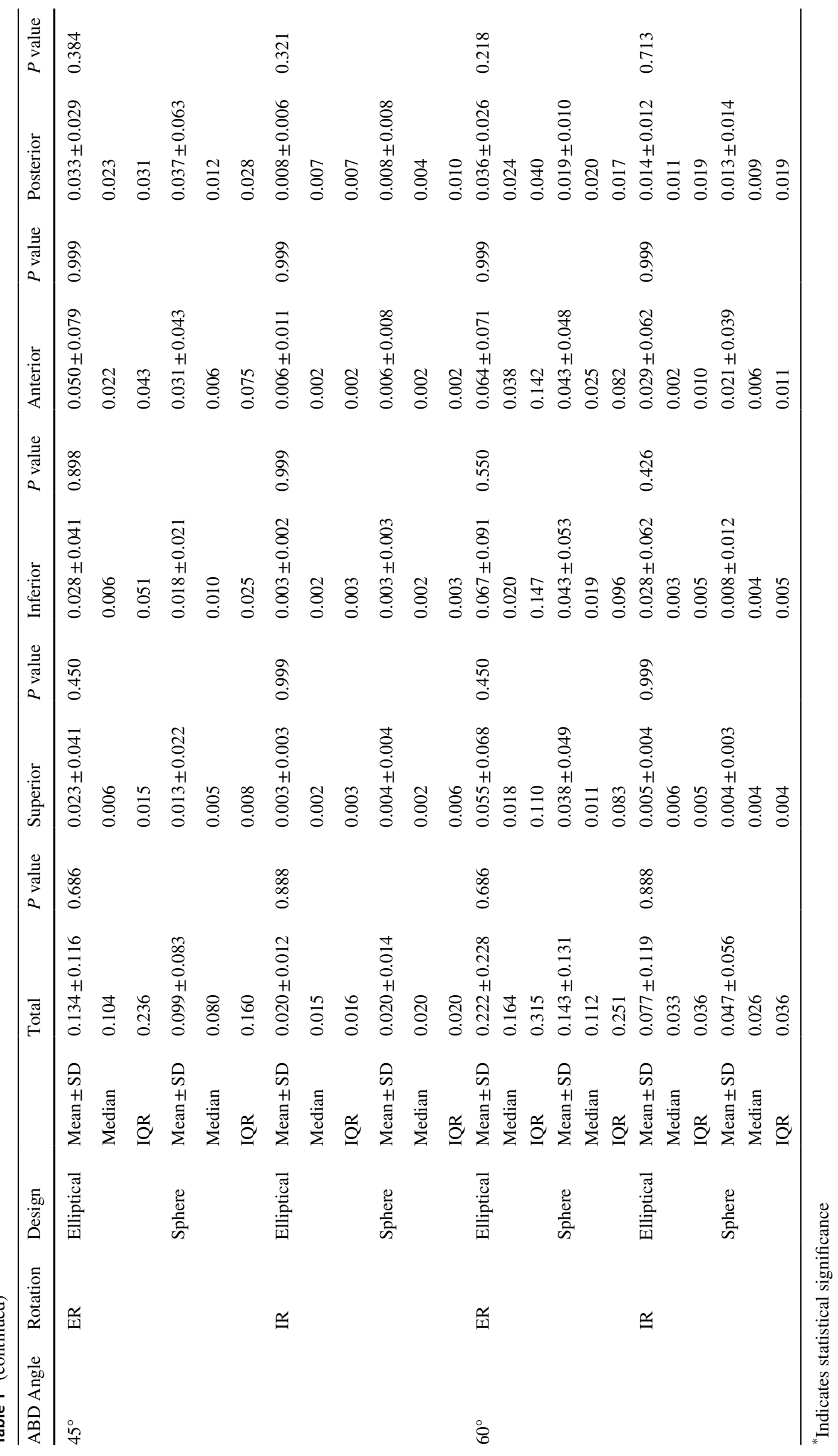


During internal rotation, elliptical and spherical heads showed similar amounts of micro-motion at the glenoid component at all tested abduction angles (Table 1). Additionally, there were no significant differences in micromotion for both elliptical and spherical heads when comparing the resting position $\left(15^{\circ}\right)$ to the abduction angles of $30^{\circ}, 45^{\circ}$, and $60^{\circ}(P>0.05$, respectively $)$.

\section{Glenohumeral translation}

Elliptical and spherical heads showed similar anteroposterior and superoinferior translation as well as compound motion during external rotation at all tested abduction angles $(P>0.05$, respectively) (Table 2). In addition, there were no significant differences in translation during external rotation for both elliptical and spherical heads when comparing the resting position $\left(15^{\circ}\right)$ to the abduction angles of $30^{\circ}, 45^{\circ}$, and $60^{\circ}(P>0.05$, respectively).

During internal rotation, the elliptical design resulted in significantly more anteroposterior translation (Fig. 4) and compound motion (Fig. 5) at all abduction angles when compared to the spherical design (Table 2). However, there was no significant difference when looking at superoinferior translation. Further, the elliptical head showed significantly less anteroposterior translation $(P=0.006)$ and compound motion $(P=0.012)$ during internal rotation at $60^{\circ}$ of abduction when compared to the resting position $\left(15^{\circ}\right)$. All other comparisons for anteroposterior and superoinferior translation as well as compound motion were found to be nonsignificant $(P>0.05$, respectively).

\section{Discussion}

The most important finding of this study was that the elliptical head design resulted in significantly greater micromotion at the glenoid component during external rotation at lower abduction angles when compared to the spherical design. However, during internal rotation, elliptical and spherical heads showed similar amounts of micro-motion at all tested abduction angles. In addition, elliptical heads showed significantly more anteroposterior glenohumeral translation and overall compound motion during internal rotation at all abduction angles when compared to the spherical design. More importantly, these biomechanical time-zero findings imply that the design of the humeral head prosthesis may further influence the development of glenoid component loosening in the long-term.

In the setting of TSA, glenoid component loosening has been reported to be one of the main contributors to implant failure, with peri-glenoid radiolucent lines having been associated to poorer postoperative functional outcomes [6, $19,26]$. Eccentric loading along with the resulting rocking (micro) motion of the glenoid component has been identified as an important biomechanical factor for subsequent implant loosening, thus the glenoid design has usually been suggested to be essential for ensuring long-term stability and clinical survival [2-4]. Although biomechanical studies have shown that the glenoid design is critical for initial fixation strength [29], clinical and radiographic studies demonstrating the superiority of one design over another are yet to be reported [28, 29]. More importantly, the inconsistency of clinical findings along with recent anatomic studies describing the humeral head to be rather elliptical in shape than a perfect sphere may imply that the design of the humeral head prosthesis may also have a considerable influence on the long-term stability of the glenoid component $[8,11,13-15]$.

As implants resembling the native anatomy may ensure restoration of joint kinematics and durability most sufficiently, the use of elliptical prosthetic heads has garnered recent interest $[13,17,18,22]$. Using a dynamic shoulder model, elliptical and spherical heads were found to achieve similar amounts of total, internal, and external rotational ROM in both hemi and TSA [22]. However, Jun et al. demonstrated that non-spherical heads resulted in increased glenohumeral translation during axial rotation in the coronal, scapular, and forward elevation plane at various abduction angles when compared to spherical heads [18]. Similarly, the present study found that the elliptical design showed significantly more anteroposterior glenohumeral translation and overall compound motion during internal rotation at all abduction angles.

While this increased translation resembles native glenohumeral kinematics more accurately, $[17,18]$ this may also lead to more eccentric loading on and greater micro-motion of the glenoid component. In this study, the elliptical head design demonstrated significantly greater micro-motion at the glenoid component during external rotation when compared to the spherical design. This was especially observed at lower abduction angles, where the glenohumeral joint is less constraint. As excessive micro-motion of the glenoid component has been suggested as a significant risk factor for glenoid loosening in the long-term, [2-4, 29] these biomechanical time-zero findings may be of clinical importance.

However, transferability into the clinical setting may be limited, as rotator cuff muscles and capsule were completely resected, to allow for accurate placement of the strain gauges directly on the glenoid. In presence of an intact rotator cuff and capsule, the spherical head may also be subjected to more translation, as it contains more physical material in the anteroposterior dimension compared to an elliptical head $[13,14]$. Tightening of the anterior part (during external rotation) or posterior part (during internal rotation) of the capsule may push the spherical head more posteriorly or anteriorly during axial rotation $[9$, 
Table 2 Anteroposterior and superoinferior glenohumeral translation $(\mathrm{mm})$ as well as overall compound motion (mm) during external (ER) and internal (IR) rotation at various abduction (ABD) angles

\begin{tabular}{|c|c|c|c|c|c|c|c|c|c|}
\hline ABD Angle & Rotation & Design & & Anteroposterior (mm) & $P$ value & Superoinferior (mm) & $P$ value & $\begin{array}{l}\text { Compound } \\
\text { motion } \\
(\mathrm{mm})\end{array}$ & $P$ value \\
\hline \multirow[t]{12}{*}{$15^{\circ}$} & \multirow[t]{6}{*}{ ER } & \multirow[t]{3}{*}{ Elliptical } & Mean \pm SD & $-5.1 \pm 3.3$ & \multirow[t]{6}{*}{0.196} & $0.6 \pm 1.3$ & \multirow[t]{6}{*}{0.888} & $5.3 \pm 3.2$ & \multirow[t]{6}{*}{0.189} \\
\hline & & & Median & -5.4 & & -0.1 & & 5.9 & \\
\hline & & & IQR & 3.3 & & 1.3 & & 3.2 & \\
\hline & & \multirow[t]{3}{*}{ Sphere } & Mean \pm SD & $-4.0 \pm 2.0$ & & $0.5 \pm 0.8$ & & $4.2 \pm 1.8$ & \\
\hline & & & Median & -4.8 & & 0.0 & & 4.8 & \\
\hline & & & IQR & 2.2 & & 1.2 & & 2.4 & \\
\hline & \multirow[t]{6}{*}{ IR } & \multirow[t]{3}{*}{ Elliptical } & Mean \pm SD & $7.7 \pm 4.4$ & \multirow[t]{6}{*}{$<0.001^{*}$} & $-0.1 \pm 0.5$ & \multirow[t]{6}{*}{0.688} & $7.8 \pm 4.3$ & \multirow[t]{6}{*}{$<0.001 *$} \\
\hline & & & Median & 7.4 & & 0.1 & & 7.4 & \\
\hline & & & IQR & 3.2 & & 0.1 & & 3.2 & \\
\hline & & \multirow[t]{3}{*}{ Sphere } & Mean \pm SD & $6.0 \pm 4.4$ & & $0.0 \pm 0.1$ & & $6.1 \pm 4.4$ & \\
\hline & & & Median & 5.1 & & 0.0 & & 5.1 & \\
\hline & & & IQR & 2.7 & & 0.1 & & 2.7 & \\
\hline \multirow[t]{12}{*}{$30^{\circ}$} & \multirow[t]{6}{*}{ ER } & \multirow[t]{3}{*}{ Elliptical } & mean $\pm \mathrm{SD}$ & $-5.0 \pm 3.9$ & \multirow[t]{6}{*}{0.180} & $0.6 \pm 1.4$ & \multirow[t]{6}{*}{0.980} & $5.2 \pm 3.9$ & \multirow[t]{6}{*}{0.212} \\
\hline & & & Median & -3.9 & & 0.1 & & 3.9 & \\
\hline & & & IQR & 7.6 & & 1.0 & & 7.3 & \\
\hline & & \multirow[t]{3}{*}{ Sphere } & Mean \pm SD & $-4.0 \pm 3.0$ & & $0.6 \pm 1.5$ & & $4.2 \pm 3.0$ & \\
\hline & & & Median & -4.2 & & 0.1 & & 4.2 & \\
\hline & & & IQR & 3.6 & & 0.5 & & 4.6 & \\
\hline & \multirow[t]{6}{*}{ IR } & \multirow[t]{3}{*}{ Elliptical } & Mean \pm SD & $8.2 \pm 3.1$ & \multirow[t]{6}{*}{$<0.001 *$} & $0.5 \pm 0.9$ & \multirow[t]{6}{*}{0.082} & $8.3 \pm 3.0$ & \multirow[t]{6}{*}{$<0.001 *$} \\
\hline & & & Median & 7.4 & & 0.1 & & 7.4 & \\
\hline & & & IQR & 2.9 & & 0.7 & & 2.9 & \\
\hline & & \multirow[t]{3}{*}{ Sphere } & Mean \pm SD & $6.0 \pm 3.9$ & & $0.0 \pm 0.1$ & & $6.0 \pm 3.9$ & \\
\hline & & & Median & 5.7 & & 0.0 & & 5.7 & \\
\hline & & & IQR & 3.0 & & 0.1 & & 3.0 & \\
\hline $45^{\circ}$ & ER & Elliptical & Mean \pm SD & $-4.3 \pm 3.1$ & 0.395 & $0.2 \pm 0.6$ & 0.666 & $4.4 \pm 3.0$ & 0.379 \\
\hline & & & Median & -4.1 & & 0.4 & & 4.2 & \\
\hline & & & IQR & 2.8 & & 0.6 & & 2.8 & \\
\hline & & Sphere & Mean \pm SD & $-3.7 \pm 2.8$ & & $0.1 \pm 0.5$ & & $3.8 \pm 2.7$ & \\
\hline & & & Median & -3.3 & & 0.0 & & 3.4 & \\
\hline & & & IQR & 2.7 & & 0.1 & & 2.7 & \\
\hline & IR & Elliptical & Mean \pm SD & $6.9 \pm 3.6$ & $0.004 *$ & $0.0 \pm 0.2$ & 0.910 & $6.9 \pm 3.6$ & $0.008 *$ \\
\hline & & & Median & 7.1 & & 0.0 & & 7.1 & \\
\hline & & & IQR & 2.3 & & 0.2 & & 2.3 & \\
\hline & & Sphere & Mean \pm SD & $5.8 \pm 3.5$ & & $0.0 \pm 0.1$ & & $5.8 \pm 3.5$ & \\
\hline & & & Median & 5.9 & & 0.0 & & 5.9 & \\
\hline & & & IQR & 2.9 & & 0.2 & & 2.9 & \\
\hline
\end{tabular}


Table 2 (continued)

\begin{tabular}{|c|c|c|c|c|c|c|c|c|c|}
\hline ABD Angle & Rotation & Design & & Anteroposterior (mm) & $P$ value & Superoinferior $(\mathrm{mm})$ & $P$ value & $\begin{array}{l}\text { Compound } \\
\text { motion } \\
(\mathrm{mm})\end{array}$ & $P$ value \\
\hline \multirow[t]{12}{*}{$60^{\circ}$} & \multirow[t]{6}{*}{ ER } & \multirow[t]{3}{*}{ Elliptical } & Mean \pm SD & $-4.1 \pm 2.2$ & \multirow[t]{6}{*}{0.240} & $0.7 \pm 0.7$ & \multirow[t]{6}{*}{0.651} & $4.2 \pm 2.3$ & \multirow[t]{6}{*}{0.252} \\
\hline & & & Median & -4.6 & & 0.7 & & 4.6 & \\
\hline & & & IQR & 2.1 & & 1.2 & & 2.2 & \\
\hline & & \multirow[t]{3}{*}{ Sphere } & Mean \pm SD & $-3.2 \pm 1.9$ & & $0.5 \pm 0.7$ & & $3.3 \pm 1.9$ & \\
\hline & & & Median & -3.2 & & 0.2 & & 3.3 & \\
\hline & & & IQR & 3.4 & & 0.8 & & 3.3 & \\
\hline & \multirow[t]{6}{*}{ IR } & \multirow[t]{3}{*}{ Elliptical } & Mean \pm SD & $6.4 \pm 3.3$ & \multirow[t]{6}{*}{$0.004^{*}$} & $0.5 \pm 0.9$ & \multirow[t]{6}{*}{0.867} & $6.4 \pm 3.4$ & \multirow[t]{6}{*}{$0.008 *$} \\
\hline & & & Median & 6.2 & & 0.1 & & 6.2 & \\
\hline & & & IQR & 2.6 & & 0.1 & & 3.0 & \\
\hline & & \multirow[t]{3}{*}{ Sphere } & $\mathrm{MEAN} \pm \mathrm{SD}$ & $5.2 \pm 3.1$ & & $0.4 \pm 0.9$ & & $5.3 \pm 3.1$ & \\
\hline & & & Median & 5.4 & & 0.1 & & 5.4 & \\
\hline & & & IQR & 2.7 & & 0.1 & & 3.1 & \\
\hline
\end{tabular}

*Indicates statistical significance. Negative values indicate posterior or superior translation, respectively
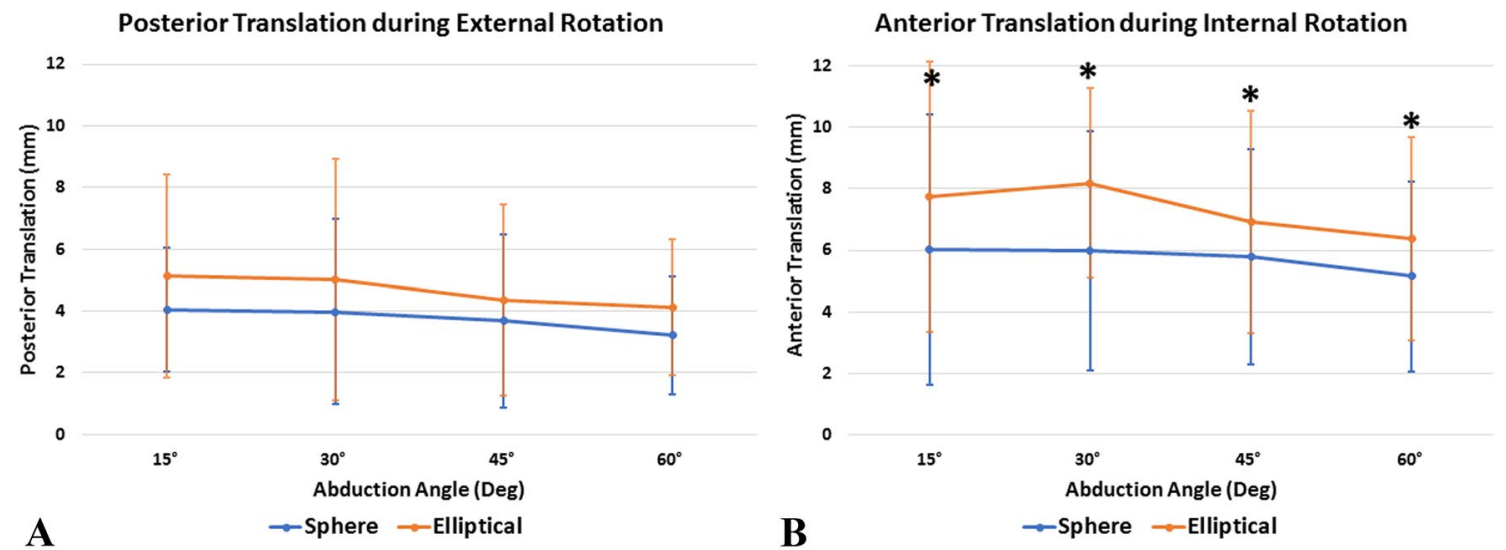

Fig. 4 Amount of glenohumeral translation $(\mathrm{mm})$ in the posterior direction during external rotation $(\mathbf{A})$ and in the anterior direction during internal rotation $(\mathbf{B})$. * Indicates statistical significance
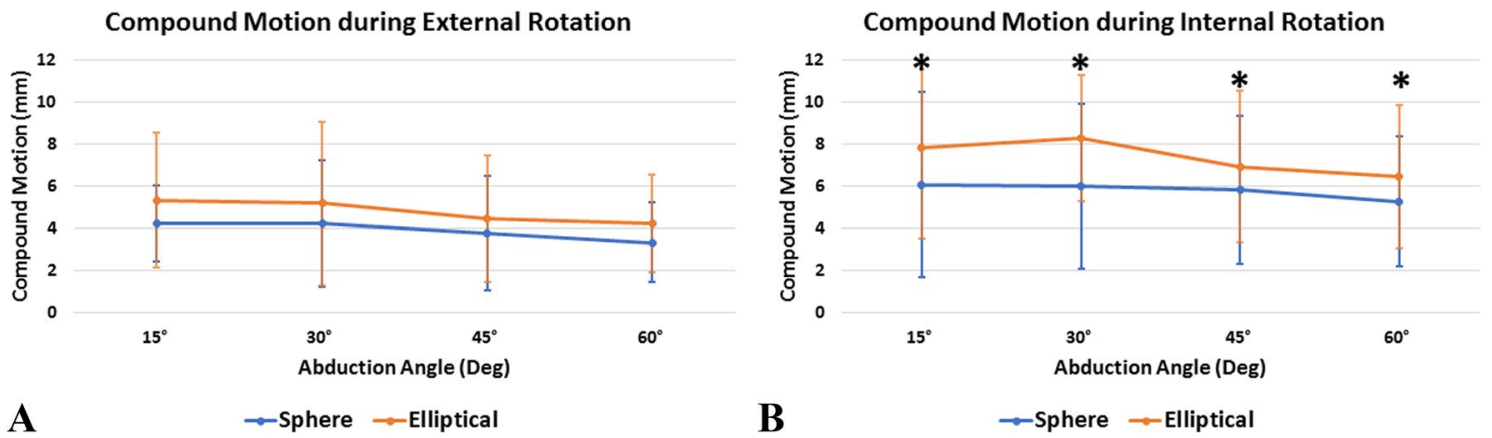

Fig. 5 Amount of overall compound motion ( $\mathrm{mm}$ ) during external (A) and internal (B) rotation. * Indicates statistical significance 
12]. This may especially be observed at higher abduction angles with the glenohumeral joint being more constraint.

To date, clinical data regarding functional outcomes and long-term stability of shoulder arthroplasty using nonspherical humeral head implants are limited. Recently, Egger et al. reported no signs of component loosening along with significant improvement of shoulder function following non-spherical TSA [5]. However, the mean follow-up was only 43 months, which does not allow for drawing definite conclusions regarding implant longevity, and there was no control group of patients undergoing TSA using a spherical design [5]. Given the various morphologies of the humeral head, especially in the setting of osteoarthritis [8], may make the availability of both head designs important for functional outcomes.

There were several limitations to the study. Humeral head prosthetic design may show a different effect in vivo when compared to observations during laboratory cadaveric testing. In addition, the study is limited to the prior resection of rotator cuff muscles and capsuloligamentous structures, leaving the effect of these soft tissue restraints on glenohumeral translation and micro-motion unknown. As such, dynamic and static glenohumeral stabilizers including the negative intraarticular pressure and subsequent joint concavity compression were eliminated, usually having further implications on glenohumeral translation. However, removal of soft tissue was essential for accurate placement of the highly sensitive strain gauges, ensuring correct measurements without disruptive factors. Lastly, the inconsistencies in the anatomy of each individual specimen, with the humeral head either being more elliptical or spherical in shape, may have further influenced the results.

\section{Conclusion}

With the use of an elliptical head design in TSA, significantly greater micro-motion at the glenoid component during external rotation at lower abduction angles can be expected when compared to the spherical design. Further, elliptical heads showed significantly more anteroposterior glenohumeral translation and overall compound motion during internal rotation at all abduction angles, potentially influencing glenoid component loosening over time.

Funding Open Access funding enabled and organized by Projekt DEAL. The University of Connecticut Health Center/UConn Musculoskeletal Institute receives funding from Arthrex Inc. (Naples, FL, USA). The company had no influence on the study design, data collection, or interpretation of the results of the final article.

\section{Declarations}

Conflict of interest LNM received material support from Arthrex Inc. (Naples, FL, USA) while conducting the study. ADM receives consulting fees as well as research support from Arthrex Inc. (Naples, FL, USA). MPC reports personal fees outside the submitted work from the Arthroscopy Association of North America. All remaining authors declare no conflict of interest.

Ethical approval This article does not contain any studies with human participants or animals performed by any of the authors. As de-identified specimens were not considered to constitute human subjects research, prior Institutional Review Board approval was not required.

Informed consent Not applicable.

Open Access This article is licensed under a Creative Commons Attribution 4.0 International License, which permits use, sharing, adaptation, distribution and reproduction in any medium or format, as long as you give appropriate credit to the original author(s) and the source, provide a link to the Creative Commons licence, and indicate if changes were made. The images or other third party material in this article are included in the article's Creative Commons licence, unless indicated otherwise in a credit line to the material. If material is not included in the article's Creative Commons licence and your intended use is not permitted by statutory regulation or exceeds the permitted use, you will need to obtain permission directly from the copyright holder. To view a copy of this licence, visit http://creativecommons.org/licenses/by/4.0/.

\section{References}

1. Arciero RA, Parrino A, Bernhardson AS et al (2015) The effect of a combined glenoid and Hill-Sachs defect on glenohumeral stability: a biomechanical cadaveric study using 3-dimensional modeling of 142 patients. Am J Sports Med 43(6):1422-1429

2. Bohsali KI, Wirth MA, Rockwood CA (2006) Complications of total shoulder arthroplasty. J Bone Joint Surg Am 88(10):2279-2292

3. Chin PY, Sperling JW, Cofield RH, Schleck C (2006) Complications of total shoulder arthroplasty: are they fewer or different? J Shoulder Elbow Surg 15(1):19-22

4. Collins D, Tencer A, Sidles JA, Matsen FA (1992) Edge displacement and deformation of glenoid components in response to eccentric loading. The effect of preparation of the glenoid bone. J Bone Joint Surg Am 74(4):501-507

5. Egger AC, Peterson J, Jones MH, Miniaci A (2019) Total shoulder arthroplasty with nonspherical humeral head and inlay glenoid replacement: clinical results comparing concentric and nonconcentric glenoid stages in primary shoulder arthritis. JSES Open Access 3(3): 145-153

6. Fox TJ, Foruria AM, Klika BJ, Sperling JW, Schleck CD, Cofield RH (2013) Radiographic survival in total shoulder arthroplasty. J Shoulder Elbow Surg 22(9):1221-1227

7. Habermeyer P, Lichtenberg S, Tauber M, Magosch P (2015) Midterm results of stemless shoulder arthroplasty: a prospective study. J Shoulder Elbow Surg 24(9):1463-1472

8. Habermeyer P, Magosch P, Weiss C et al (2017) Classification of humeral head pathomorphology in primary osteoarthritis: a radiographic and in vivo photographic analysis. J Shoulder Elbow Surg 26(12):2193-2199 
9. Harryman DT, Sidles JA, Clark JM, McQuade KJ, Gibb TD, Matsen FA (1990) Translation of the humeral head on the glenoid with passive glenohumeral motion. J Bone Joint Surg Am 72(9):1334-1443

10. Hawi N, Magosch P, Tauber M, Lichtenberg S, Habermeyer P (2017) Nine-year outcome after anatomic stemless shoulder prosthesis: clinical and radiologic results. J Shoulder Elbow Surg 26(9):1609-1615

11. Hertel R, Knothe U, Ballmer FT (2002) Geometry of the proximal humerus and implications for prosthetic design. J Shoulder Elbow Surg 11(4):331-338

12. Howell SM, Galinat BJ, Renzi AJ, Marone PJ (1988) Normal and abnormal mechanics of the glenohumeral joint in the horizontal plane. J Bone Joint Surg Am 70(2):227-232

13. Humphrey CS, Gale AL (2018) Spherical versus elliptical prosthetic humeral heads: a comparison of anatomic fit. J Shoulder Elbow Surg 27(6S):S50-S57

14. Humphrey CS, Sears BW, Curtin MJ (2016) An anthropometric analysis to derive formulae for calculating the dimensions of anatomically shaped humeral heads. J Shoulder Elbow Surg 25(9):1532-1541

15. Iannotti JP, Gabriel JP, Schneck SL, Evans BG, Misra S (1992) The normal glenohumeral relationships. An anatomical study of one hundred and forty shoulders. J Bone Joint Surg Am 74(4):491-500

16. Imhoff FB, Camenzind RS, Obopilwe E et al (2019) Glenoid retroversion is an important factor for humeral head centration and the biomechanics of posterior shoulder stability. Knee Surg Sports Traumatol Arthrosc. https://doi.org/10.1007/s00167-019-05573-5

17. Jun BJ, Iannotti JP, McGarry MH, Yoo JC, Quigley RJ, Lee TQ (2013) The effects of prosthetic humeral head shape on glenohumeral joint kinematics: a comparison of non-spherical and spherical prosthetic heads to the native humeral head. J Shoulder Elbow Surg 22(10):1423-1432

18. Jun BJ, Lee TQ, McGarry MH, Quigley RJ, Shin SJ, Iannotti JP (2016) The effects of prosthetic humeral head shape on glenohumeral joint kinematics during humeral axial rotation in total shoulder arthroplasty. J Shoulder Elbow Surg 25(7):1084-1093

19. Kilian CM, Morris BJ, Sochacki KR et al (2018) Radiographic comparison of finned, cementless central pegged glenoid component and conventional cemented pegged glenoid component in total shoulder arthroplasty: a prospective randomized study. J Shoulder Elbow Surg 27(6S):S10-S16

20. Mehl J, Otto A, Imhoff FB et al (2019) Dynamic anterior shoulder stabilization with the long head of the biceps tendon: a biomechanical study. Am J Sports Med 47(6):1441-1450
21. Mihata T, Lee Y, McGarry MH, Abe M, Lee TQ (2004) Excessive humeral external rotation results in increased shoulder laxity. Am J Sports Med 32(5):1278-1285

22. Muench LN, Otto A, Kia C et al (2020) Rotational range of motion of elliptical and spherical heads in shoulder arthroplasty: a dynamic biomechanical evaluation. Arch Orthop Trauma Surg. https://doi.org/10.1007/s00402-020-03587-0

23. Papadonikolakis A, Neradilek MB, Matsen FA 3rd (2013) Failure of the glenoid component in anatomic total shoulder arthroplasty: a systematic review of the English-language literature between 2006 and 2012. J Bone Joint Surg Am 95(24):2205-2212

24. Pauzenberger L, Dyrna F, Obopilwe E et al (2017) Biomechanical evaluation of glenoid reconstruction with an implant-free j-bone graft for anterior glenoid bone loss. Am J Sports Med 45(12):2849-2857

25. Schneider DJ, Tibone JE, McGarry MH, Grossman MG, Veneziani S, Lee TQ (2005) Biomechanical evaluation after five and ten millimeter anterior glenohumeral capsulorrhaphy using a novel shoulder model of increased laxity. J Shoulder Elbow Surg 14(3):318-323

26. Schoch BS, Wright TW, Zuckerman JD et al (2019) Glenoid component lucencies are associated with poorer patient-reported outcomes following anatomic shoulder arthroplasty. J Shoulder Elbow Surg 28(10):1956-1963

27. Selecky MT, Tibone JE, Yang BY, McMahon PJ, Lee TQ (2003) Glenohumeral joint translation after arthroscopic thermal capsuloplasty of the posterior capsule. J Shoulder Elbow Surg 12(3):242-246

28. Throckmorton TW, Zarkadas PC, Sperling JW, Cofield RH (2010) Pegged versus keeled glenoid components in total shoulder arthroplasty. J Shoulder Elbow Surg 19(5):726-733

29. Voss A, Beitzel K, Obopilwe E et al (2017) No correlation between radiolucency and biomechanical stability of keeled and pegged glenoid components. BMC Musculoskelet Disord 18(1):213

Publisher's Note Springer Nature remains neutral with regard to jurisdictional claims in published maps and institutional affiliations. 